\title{
SELF-DUAL CONNECTIONS AND THE TOPOLOGY OF SMOOTH 4-MANIFOLDS
}

\author{
S. K. DONALDSON ${ }^{1}$
}

1. Introduction, statement of result. To any compact oriented 4-manifold $X$ there is associated a quadratic form $Q$, defined on the cohomology group $H^{2}(X ; \mathbf{Z})$ by $Q(\alpha)=(\alpha \cup \alpha)[X]$. Poincaré duality requires that it be a "unimodular" form-given by a symmetric matrix of determinant \pm 1 with respect to any base for the torsion free part of $H^{2}$. It is known from arithmetic that there are many such forms that are positive definite and not equivalent (over the integers) to the standard form [4, Chapter 5]. The problem of finding which forms are realised by simply-connected 4-manifolds was raised, for example, in [3]; a partial answer for smooth 4-manifolds is announced here in the form of

THEOREM. If $X$ is a smooth, compact, simply-connected oriented 4-manifold with the property that the associated form $Q$ is positive definite, then $Q$ is equivalent, over the integers, to the standard diagonal form.

As a particular application, the theorem shows that it is impossible to remove smoothly, by surgery, all three hyperbolic factors in a K3 surface (which has quadratic form $E_{8}+E_{8}+\left(\begin{array}{ll}0 & 1 \\ 1 & 0\end{array}\right)+\left(\begin{array}{ll}0 & 1 \\ 1 & 0\end{array}\right)+\left(\begin{array}{ll}0 & 1 \\ 1 & 0\end{array}\right)$ ) since this would give a simply-connected smooth 4-manifold with definite form $E_{8}+E_{8}$.

2. Method of proof. I give, in this note, an outline of the proof; a detailed account will appear soon. The idea of the proof is to exploit topological information that emerges from a study of "self-dual connections" or "instantons"; I take [1] as a general reference for background in this area, and for notation. Suppose throughout that $X$ is a 4-manifold satisfying the hypotheses of the theorem, and that we are given some Riemannian metric.

There is, up to isomorphism, a unique principal $S U(2)$ bundle $P$ over $X$ with characteristic class $c_{2}(P)[X]=-1$. One forms the space of all equivalence classes of connections on $P$ as the quotient of the affine space $A$ of connections by the action of the "gauge group" $\mathcal{G}$ of automorphisms of $P$. A Hausdorff topology descends to $A / \mathcal{G}$ and the dense open subset representing irreducible connections can be made into a Banach manifold. On the other hand a reducible connection corresponds to a reduction of $P$ to an $S^{1}$ bundle and in the neighbourhood of such a point the space $A / \mathcal{G}$ has the structure of

(Real Banach Space) $\times\left(\right.$ Complex Banach Space $\left./ S^{1}\right)$.

Received by the editors August 9, 1982.

1980 Mathematics Subject Classification. Primary 57N13; Secondary 58G99.

${ }^{1}$ Work supported by the Science and Engineering Research Council of the United Kingdom.

(C) 1983 American Mathematical Society 0273-0979/82/0000-1037/\$01.75 
The $S^{1}$ action arises as the action of the stabiliser in $\mathcal{G}$ of a reducible connection.

A simple calculation with characteristic classes shows that the number of topologically distinct reductions of $P$, and so the number of components of the singular subset of $A / \mathcal{G}$, is given in terms of the form $Q$ by

$$
n(Q)=\frac{1}{2} \#\left\{\alpha \in H^{2}(X ; \mathbf{Z}): Q(\alpha)=1\right\} \text {. }
$$

3. Self-dual connections. A connection of $P$ is called "self-dual" if its curvature $\Omega$ satisfies the equation ${ }^{*} \Omega=\Omega$. This property is invariant under $\mathcal{G}$ so we may define the moduli space $\mathcal{M} \subset A / \mathcal{G}$ of equivalence classes of self-dual connections on $P$.

Atiyah, Hitchin, and Singer $[1, \S 6]$ showed that, provided a certain cohomology group vanishes, the irreducible elements of $\mathcal{M}$ form a smooth finitedimensional submanifold of $A / \mathcal{G}$. Their formula for the dimension gives in the special case at hand

$$
\operatorname{dim} \mathcal{M}=8-\left(\frac{3}{2}\right)(\chi(X)-\tau(X))=5 .
$$

In general we do not know that the vanishing condition will be satisfied, but this is not important for the topological application since one may show

LEMMA 1. For suitable generic perturbations of the self-duality equations the perturbed moduli space $\mathcal{M}^{*} \subset \mathcal{A} / \mathcal{G}$ is a smooth 5-manifold at all points representing irreducible connections.

This is a straightforward extension of a standard general position argument to an infinite-dimensional setting; using the methods of Kuranishi [2], or of Smale [5].

It is not obvious that there are any self-dual connections on $P$ at all. Their existence follows from a recent theorem of C. H. Taubes [6, Theorem 1.2], once one observes that an equivalent form of the hypothesis that the quadratic form be positive is the statement that all harmonic 2-forms on $X$ are self-dual.

Then, using the techniques of $[6]$ together with results of $\mathrm{K}$. Uhlenbeck [7, 8 ], and guided by what is known for the case when $X=S^{4}[1]$, one has a good understanding of the "boundary" of $\mathcal{M}$.

LEMMA 2. There is an open subset $\mathcal{U} \subset \mathcal{M}$ which is a smooth 5-manifold diffeomorphic to $X \times(0,1)$, and $\mathcal{M} \backslash \mathcal{U}$ is compact.

Notes. (i) In proving this lemma I use the fact that $X$ is simply connected.

(ii) The perturbed moduli space $\mathcal{M}^{*}$ of Lemma 1 has the same boundary properties as $\mathcal{M}$.

At each reducible connection in $\mathcal{M}^{*}$ the 5 -manifold inherits a quotient singularity from the ambient space; around such a point, $\mathcal{M}^{*}$ has the structure of $\mathbf{C}^{3} / S^{1}$ : a cone on $\mathbf{C P}^{2}$. $\mathcal{M}$, and so $\mathcal{M}^{*}$, meets each component of the reducible connections in $A / \mathcal{G}$ exactly once, so there are $n(Q)$ singular points. Thus there is a smooth compact 5-manifold with boundary the disjoint union of $X$ and $n(Q)$ copies of $\mathbf{C P}^{2}$.

Finally, an argument based on properties of the index for families of operators shows that this 5 -manifold is orientable. (This was first proved by M. F. Atiyah.) 
4. Proof of theorem. Note first that for any unimodular positive form $Q$, $n(Q) \leq \operatorname{rank}(Q)$ with equality if and only if $Q$ is equivalent to the standard form.

Then apply the fact that signature is an invariant of oriented cobordism to the 5-manifold above. The signature of $X$ is $\operatorname{rank}(Q)$ so there must be at least $\operatorname{rank}(Q)$ copies of $\mathbf{C P}^{2}$. Hence $n(Q)=\operatorname{rank}(Q)$ and $Q$ is equivalent to the standard diagonal form.

\section{REFERENCES}

1. M. F. Atiyah, N. J. Hitchin and I. M. Singer, Self-duality in 4-dimensional Riemannian geometry, Proc. Roy. Soc. London Ser. A 362 (1978), 425-461.

2. M. Kuranishi, New proof of the existence of locally complete families of complex structures, Proc. Conf. Complex Analysis (Aeppi et al, (eds.)), Springer-Verlag, Berlin and New York, 1964, pp. 142-154.

3. J. Milnor, On simply connected 4-manifolds, Internat. Sympos. Algebraic Topology (Mexico, 1958).

4. J-P. Serre, $A$ course in arithmetic, Springer-Verlag, Berlin and New York, 1973.

5. S. Smale, An infinite dimensional version of Sard's Theorem, Amer. J. Math. 87 (1965), 861-866.

6. C. H. Taubes, The existence of self-dual connections on non self-dual 4-manifolds, J. Differential Geom. (to appear).

7. K. K. Uhlenbeck, Connections with $L^{p}$ bounds on curvature, Comm. Math. Phys. 3 (1981).

8. __ Removable singularities in Yang-Mills fields, Comm. Math. Phys. 3 (1981).

The Mathematical Institute, 24-29 St. Giles, OXford, England 